\title{
Aerodynamic Performance of the Three-Dimensional Lumped Flexibility Bionic Hovering Wing
}

\author{
Zhu Jianyang, Jiang Lin (D), and Hou Yu \\ School of Machinery and Automation, Wuhan University of Science and Technology, Wuhan 430081, China \\ Correspondence should be addressed to Jiang Lin; jlxyhjl@163.com
}

Received 18 October 2017; Revised 12 February 2018; Accepted 28 February 2018; Published 10 April 2018

Academic Editor: Linda L. Vahala

Copyright (C) 2018 Zhu Jianyang et al. This is an open access article distributed under the Creative Commons Attribution License, which permits unrestricted use, distribution, and reproduction in any medium, provided the original work is properly cited.

\begin{abstract}
Understanding the effect of flexibility on the aerodynamic characteristics of the wing is one of the most important considerations for successfully designing a flapping wing micro aero vehicle (FMAV). This paper aims at providing a systematic synthesis on the flexibility effects on the hovering performance of the bionic wing based on the numerical analysis approach. We construct a novel three-dimensional bionic wing, which has lumped flexibility at the root, and develop an iterative coupling program to simulate the interaction between the flexible wing and fluid. The effects of flexibility on the hovering performance of the three-dimensional flapping wing are investigated, and the results indicate that the best performance of the wing is achieved when the wing flaps at resonance and has the density close to the natural insect wing. The feasibility of using lumped flexibility wings driven by a simple harmonic flapping for designing efficient FMAV is also concluded in this study.
\end{abstract}

\section{Introduction}

Flapping wing aero vehicle has become a popular research topic in recent years because it has better aerodynamic performance than the fixed-wing and rotary-wing aero vehicle under low Reynolds number flight regime [1-3]. Understanding the effect of flexibility on the aerodynamic characteristics of the wing is one of the most important considerations for successfully designing this type of vehicle. Therefore, many studies have been carried out to investigate this problem, and considerable understandings have been achieved [4-7].

The lumped flexibility wing for insect flight was first proposed by Ishihara et al. in [8]. In their study, the twodimensional wing was modeled by a rectangular plate, and the wing's flexibility was lumped into the spring at the leading edge. It was found that the studied wing can generate sufficient lift force to support the insect's weight. Therefore, they concluded that the pitching motion can be passive in the dipteran's flapping flight. Later, they again experimentally and numerically $[9,10]$ investigated the performance of the three-dimensional lumped flexible wing, and it was also found that if the wing has appropriate flexibility, the lumped flexible wing can generate sufficient lift force to support the corresponding insect weight.

Michelin and Llewellyn Smith [11] studied the influence of the flexibility on the performance of a heaving wing. They found that the maximum values of the mean thrust and efficiency are obtained when the resonance occurs between the forcing frequency and a natural frequency of the system. Masoud and Alexeev [12] conducted a numerical study to focus on the aerodynamic characteristics of a flexible planar hovering wing which was flapped at resonance. They again concluded that large-amplitude resonance flapping wing can drastically enhance aerodynamic lift and efficiency of the wing under low Reynolds number. Moore [13] also found that dramatic performance improves when the wing is driven near resonance and deteriorates when it is driven at higher frequencies. Zhu et al. [14, 15] studied the aerodynamic performance of a $2 \mathrm{D}$ flexible wing in forward flight by solving the N-S equations coupled with structural dynamic equation and the flapping motion of the wing. They concluded that if the wing flapped at a frequency close but below the wing's natural vibration 


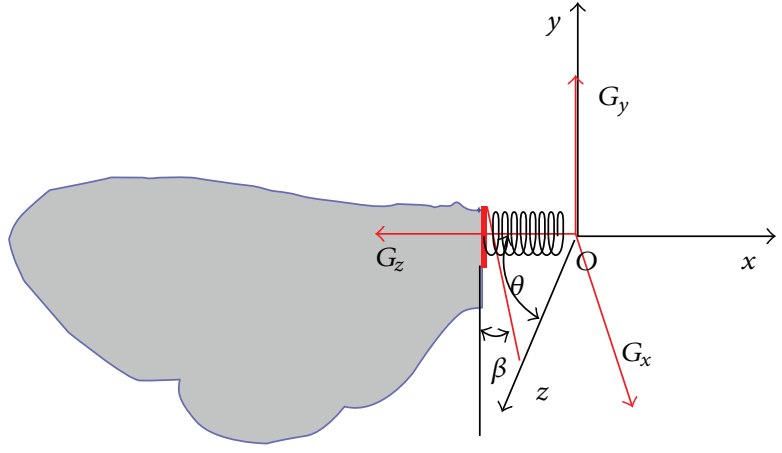

(a)

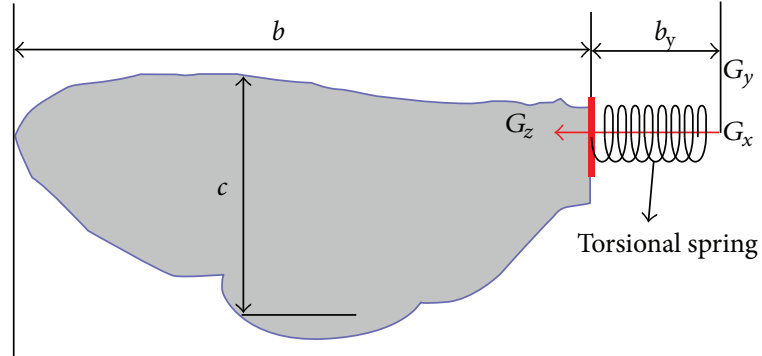

(b)

FIGURE 1: The schematic of the wing planform.

frequency, the flexibility can increase the aerodynamic force and the energy efficiency.

On the other hand, Chen et al. [16] performed a study to investigate the aerodynamic performance of a flapping and passively rotating insect wing by using experimental and computational methods. A novel wing which had a single flexible hinge allowing passive pitching under load was devised in their study. The results indicated that the wing's kinematic and flapping efficiency depends on the stiffness of a passive compliant hinge. It was not the resonance wing but the stiffness wing which had larger lift force production. Cho et al. [17] developed a computational framework to simulate a three-dimensional flapping flexible wing under simultaneous pitching and plunging motion, and the results indicated that the flexibility can be beneficial for the wing with moderate flexibility. Yeh and Alexeev [18] carried out a numerical study to investigate the performance of the plunging elastic plates with aspect ratios ranging from 0.5 to 5 . They showed that the maximum velocity occurs near the first natural frequency regardless of aspect ratio, whereas the maximum economy occurs away from the first natural frequency.

In summary, the flexibility is beneficial for the flapping wing as shown in the above literatures; however, a full understanding on the mechanism of how the flexibility affects the aerodynamic characteristics of the wing is still not achieved, especially the best performance of the wing flapping at resonance or not. To fill in these gaps, a novel three-dimensional bionic wing which has lumped flexibility is constructed, and an iterative coupling program to simulate the interaction between the flexible wing and fluid is developed. Then the details of the lift force, hovering efficiency, and vortex of the flexible bionic wing are studied.

\section{The Novel Three-Dimensional Bionic Wing Model}

Bumblebee is one of the typical insects which have amazing flying capabilities. The shape, structure, and aerodynamic characteristics of this insect's wing have been studied extensively [19-21]. In this paper, the planform of the bionic bumblebee wing is selected similar to the experimental model studied by Nagai et al. [22] for comparison. The schematic of the wing planform is shown in Figure 1, where two coordinate systems are also defined: one is the inertial coordinate system (OXYZ) and the other is the wing-fixed coordinate system $\left(\mathrm{OG}_{x} \mathrm{G}_{y} \mathrm{G}_{z}\right)$. The symbol $b=0.100 \mathrm{~m}$ is the span, $b_{r}=0.024 \mathrm{~m}$ is the distance from wing base to the flapping $y$-axis, and $c$ is the reference chord which is defined as a length at two-thirds of the span location $(c=0.01575 \mathrm{~m})$. The area of the wing $S$ is fixed at a value of $2.984 \times 10^{-3} \mathrm{~m}^{2}$, and the thickness of the wing is fixed at $S /(50 b)$ for comparison.

In contrast to the previous study that consider the chordwise and spanwise flexibility of the wing, the flexibility of the wing in this paper is mimicked as the passive pitching motion by connecting a rigid wing to a torsional spring; therefore, the flexibility is just lumped at the root of the wing, and the simplified wing is in reasonable agreement with the natural insect wing [23].

The flapping motion $\theta$ of the wing which is defined as the angle between the axis $G_{x}$ and the $z$-axis follows the simple sinusoidal function:

$$
\theta(t)=\frac{\theta_{m}}{2} \sin (2 \pi f t),
$$

where $\theta_{m}$ is the flapping amplitude and $f$ is the flapping frequency.

The passive pitching angle $\beta$ which is defined as the angle between the axis $G_{y}$ and the $y$-axis can be decided by the equation

$$
J \ddot{\beta}+K \beta=Q,
$$

where $K$ is the torsion stiffness, $Q$ the pitching torque generated by the aerodynamic force, and $J$ is the inertia moment of the wing which can be defined as

$$
J=\sum_{i} \Delta m_{i} r_{i}^{2}
$$

where $m_{i}$ is the element mass and $r_{i}$ is the distance from the element mass to the pitching axis $G_{x}$. 


\section{Parameters of Bionic Hovering Wing}

To clearly elaborate the aerodynamic characteristics of the lumped flexibility wing, three dimensionless parameters are defined, namely, the Reynolds number Re, density ratio $\rho^{*}$, and frequency ratio $f^{*}$. They can be defined as

$$
\begin{aligned}
\operatorname{Re} & =\frac{U_{\mathrm{ref}} c}{v}, \\
\rho^{*} & =\frac{\rho_{s}}{\rho}, \\
f^{*} & =\frac{f}{f_{s}},
\end{aligned}
$$

where $U_{\text {ref }}=\pi f \theta_{m}\left(2 b / 3+b_{r}\right)$ is the maximum of flapping velocity, $v$ is the fluid kinematic viscosity, $\rho$ is the fluid density, $\rho_{s}$ is the wing density, and $f_{s}$ is the wing vibration frequency which can be calculated as

$$
f_{s}=\frac{\sqrt{K / J}}{2 \pi} .
$$

Note that the fluid field characteristics are determined by Re and the flexibility of the wing is characterized by $f^{*}$ and $\rho^{*}$.

The coefficient of lift, drag, and energy consumption are described as follows:

$$
\begin{aligned}
& C_{L}=\frac{F_{L}}{0.5 \rho U_{\mathrm{ref}}^{2} S}, \\
& C_{D}=\frac{F_{D}}{0.5 \rho U_{\mathrm{ref}}^{2} S}, \\
& C_{P}=\frac{Q_{\mathrm{Y}} \dot{\theta(t)}}{0.5 \rho U_{\mathrm{ref}}^{3} S},
\end{aligned}
$$

where $F_{L}$ is the lift, $F_{D}$ the drag, $Q_{y}$ the aerodynamic torque of wing about the $y$-axis, and $\theta(t)^{\bullet}$ is the derivative of $\theta(t)$. The hovering efficiency $\eta$ then can be described as

$$
\eta=\frac{\overline{C_{L}}}{\overline{C_{P}}}
$$

where $\overline{C_{L}}$ and $\overline{C_{P}}$ are the mean lift and energy coefficient, respectively.

\section{The Coupling Program to Simulate the Interaction between Fluid and Flexible Wing}

Considering the bumblebee's flight environment in nature, the Reynolds number of the hovering wing is at the range of 102-104; therefore, the flow field of the wing is assumed to be unsteady, laminar, and incompressible with constant thermophysical properties, and the governing equations of this flow field are described by the 3D unsteady incompressible laminar N-S equations as follows:

$$
\begin{aligned}
\nabla \cdot V & =0, \\
\frac{\partial V}{\partial t}+V \cdot \nabla V & =-\nabla P+\frac{1}{\operatorname{Re}} \nabla^{2} V,
\end{aligned}
$$

where $V$ is the nondimensional fluid velocity and $P$ is the nondimensional fluid pressure.

To solve (8), we implement a numerical solver (Fluent 6.3) based on a finite volume method. The third-order MUSCL scheme [24] is used for spatial interpolation, and a first-order implicit algorithm is employed for time discretization. Meanwhile, the coupling between the pressure and the velocity is achieved by means of the PISO [25] algorithm. The flapping and passive pitching motion of the wing are controlled and achieved by the local remeshing dynamic mesh technique (see Fluent 6.3 Tutorial Guide).

In order to solve the governing equation of the passive pitching motion of the wing, the Newmark method is applied to discretization derivative of passive pitching angle, and then (2) can be rewritten as

$$
\begin{aligned}
\left(1+\gamma \Delta t^{2} \frac{K}{J}\right) \beta^{t+\Delta t}= & \frac{\bar{Q}^{t+\Delta t}}{J} \Delta t^{2}+\left[2-\left(\frac{1}{2}-2 \gamma+\delta\right) \Delta t^{2} \frac{K}{J}\right] \beta^{t} \\
& +\left[-1-\left(\frac{1}{2}+\gamma-\delta\right) \Delta t^{2} \frac{K}{J}\right] \beta^{t-\Delta t},
\end{aligned}
$$

where

$$
\bar{Q}^{t+\Delta t}=\gamma Q^{t+\Delta t}+\left(\frac{1}{2}-2 \gamma+\delta\right) Q^{t}+\left(\frac{1}{2}-\gamma-\delta\right) Q^{t-\Delta t},
$$

where $\beta^{t+\Delta t}, \beta^{t}$, and $\beta^{t-\Delta t}$ are the pitching angle of the next step time, current moment, and last step time, respectively. $Q^{t+\Delta t}, Q^{t}$, and $Q^{t-\Delta t}$ are the aerodynamic torque of the next step time, current moment, and last step time, respectively. $\Delta t$ is the iteration time step and $\gamma$ and $\delta$ are the control parameters, which are fixed as $\gamma=1 / 6$ and $\delta=1 / 2$.

An iterative coupling method is developed to simulate the interaction of fluid, flapping, and passive pitching motion of wing, where the fluid field based on finite volume method and passive pitching based on Newmark method are solved separately at each time step. First, the fluid field is solved, then the aerodynamic torque on the wing is obtained, and then the pitching angle of flexibility wing can be determined under the aerodynamic torque using Newmark method, which is embedded in Fluent using the UDF. In the next time step, the fluid field is solved with the new location of the wing updated by using the dynamic mesh technique. In this way, the fluid and the flapping and passive pitching motion are coupled so that the fluid-structure interaction is taken into account properly. The details of the coupling process are illustrated in Figure 2. 


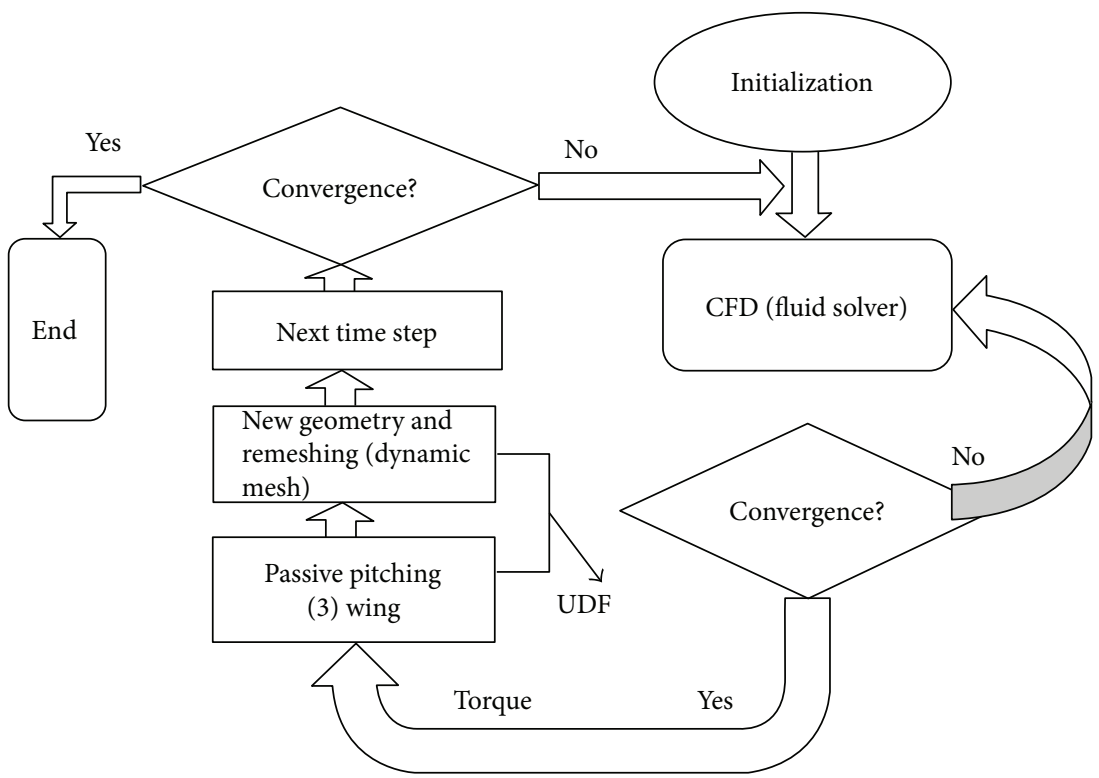

Figure 2: The schematic of coupling method.

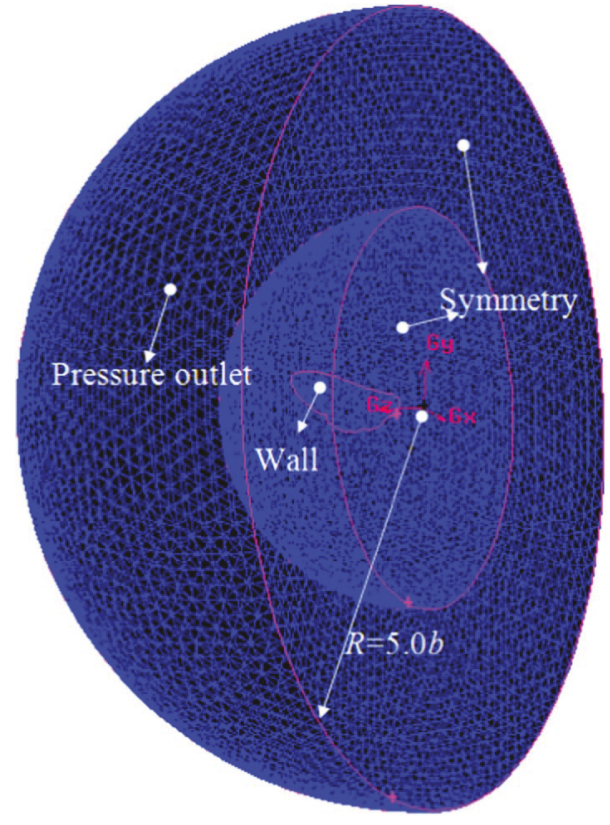

Figure 3: The computational grid and boundary conditions.

\section{Simulation Setup and Method Validation}

5.1. Simulation Setup. The computational domain has a hemispherical shape, and the radius of the hemisphere is fixed at a value of $5 b$ as shown in Figure 3. A uniform unstructured mesh system is employed, where the hemispherical shape computational domain contains an inner domain and an outer domain. Tetrahedron cells are used to cluster to the wing in the inner domain, and the density of the tetrahedron cells are decreased from the inner domain to the outer domain. The no-slip wall boundary condition is applied at the bionic hovering wing surface. Meanwhile, pressure-outlet boundary condition which has zero static pressure is imposed on the outer boundary, and symmetry boundary condition is applied on the right computational boundary to save the simulation cost.

5.2. Method Validation. A grid sensitivity study is carried out first to evaluate the independence of the numerical result on the mesh size. The unsteady flow fields of a flexible wing were computed under conditions $\rho^{*}=1000, f^{*}=1.50$, and $\operatorname{Re}=2.347 \times 10^{3}$ with three different grid systems. The sizes of involved grid systems were as follows: $3.5 \times 10^{5}$ tetrahedron cells around the wing, the first layer cell spacing above the wing $0.040 b$ (system A); $2.3 \times 10^{5}$ tetrahedron cells around the wing, the first layer cell spacing above the wing $0.060 b$ (system $\mathrm{B}$ ); and $1.9 \times 10^{5}$ tetrahedron cells around the wing, the first layer cell spacing above the wing $0.070 \mathrm{~b}$ (system C). Each grid was computed for 6 periods, and iteration time step was fixed at $0.001 T$. Only the comparison of passive pitching angle $\beta$ at different resolutions is given, because the aerodynamic performance of the developed lumped flexibility wing is mainly determined by $\beta$. The results in Figure 4 show that the difference of passive pitching angle $\beta$ of the grid systems $A$ and $B$ are very small (no more than $1 \%$ ). Therefore, grid system $B$ is sufficiently dense and it is employed for the next simulations.

Three different iteration time steps $0.05 T, 0.001 T$, and $0.0005 T(T=1 / f)$ are employed to again simulate the unsteady flow field of a flexible wing with $\rho^{*}=1000, f^{*}=$ 1.50 , and $\operatorname{Re}=2.347 \times 10^{3}$ to validate the time-discretization independence. The results in Figure 5 show that the difference of the passive pitching angle of the three iteration time steps are very small (no more than $2 \%$ ), which indicates that the iteration time step with $t=0.001 T$ is sufficiently small, and it is employed for the next simulations. 


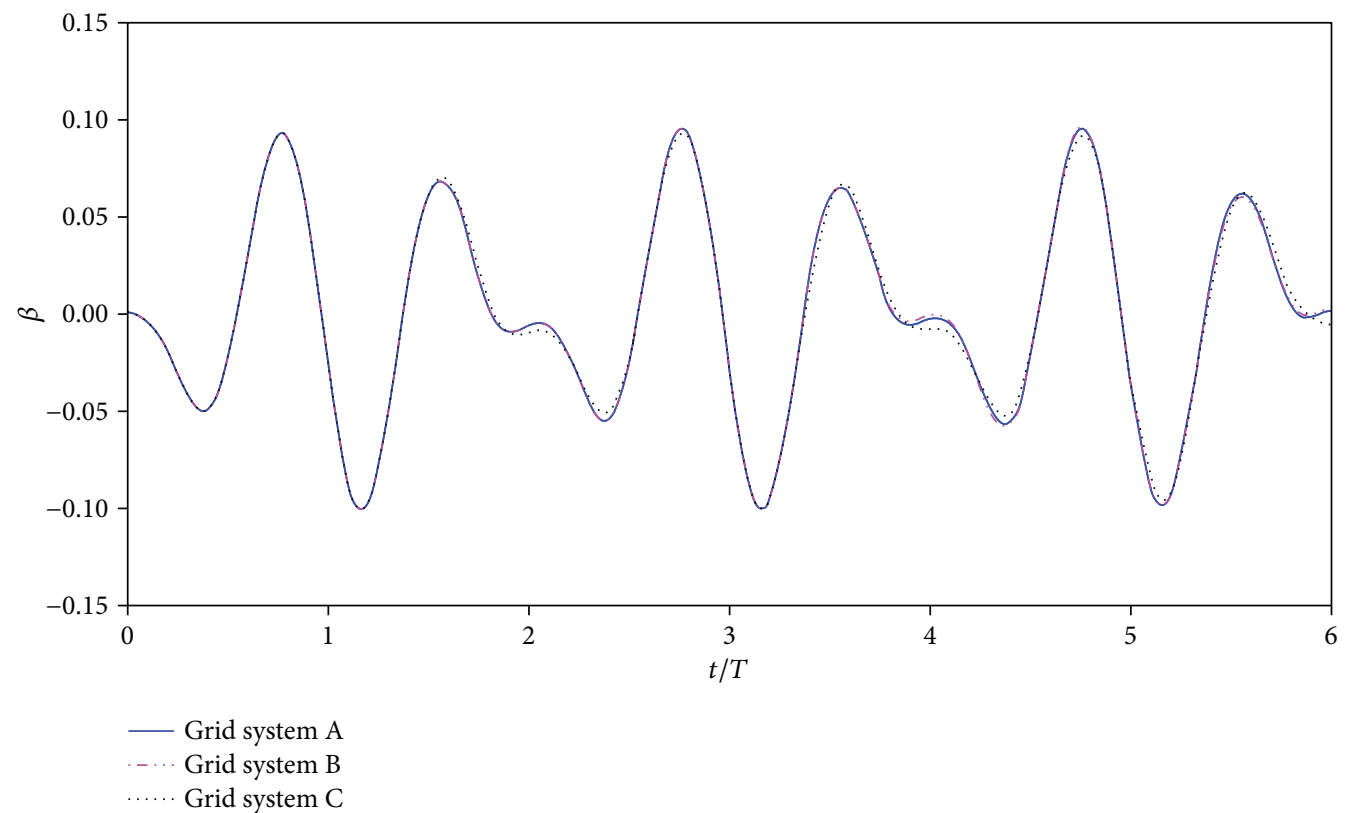

FIgURE 4: The time variation of passive pitching angle $\beta$ under three different grid systems.

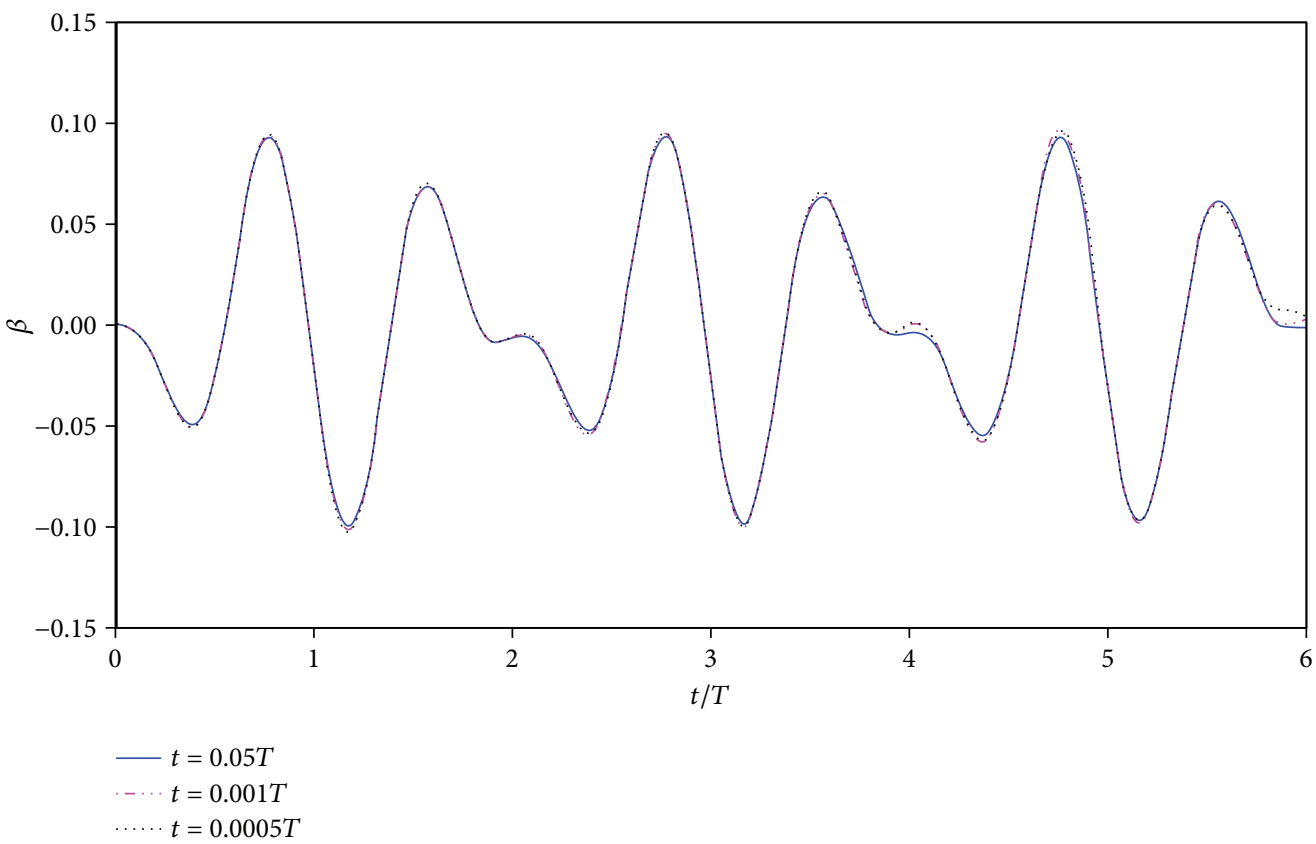

FIgURE 5: The time variation of passive pitching angle $\beta$ under three different iteration time steps.

A typically bionic bumblebee hovering wing which was experimentally studied by Nagai et al. [22] is employed to validate the reliability of the present numerical method for simulating the unsteady flow around 3D hovering wing. The mean drag and lift coefficients are -0.00 and 0.46 , respectively, in the present numerical computation, and in Nagai et al.'s experimental study, these two data are 0.00 and 0.49 , respectively. The comparison of instantaneous drag and lift coefficient are also illustrated in Figure 6. It is again found that our computational results agree with the experimental results very well.
Validation of the reliability of the developed weak coupling method for simulating the fluid-flapping wing interaction problem had been demonstrated in our previous study. The details of the validation process can be found in $[14,26]$.

\section{Results and Discussion}

To study the aerodynamic performance of the threedimensional (3D) lumped flexibility bionic hovering wing, the results of the $3 \mathrm{D}$ rigid flapping wing need to be employed 


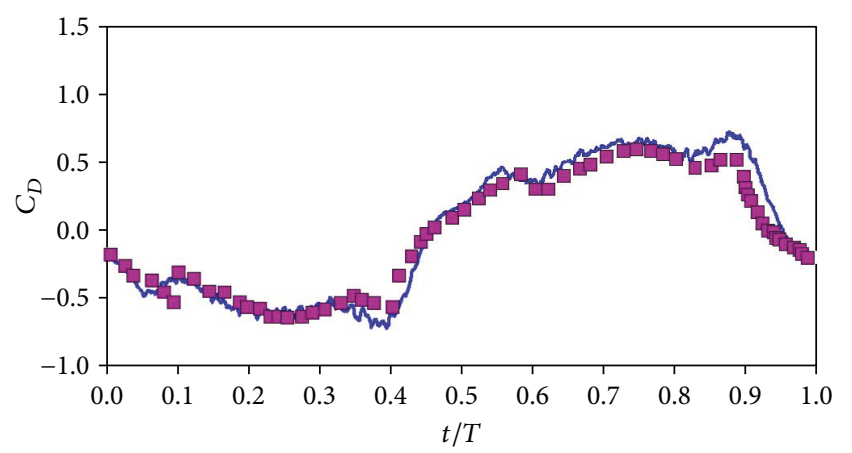

- Present results

$\square$ Experimental results by Nagai et al. [22]

(a) Drag coefficient

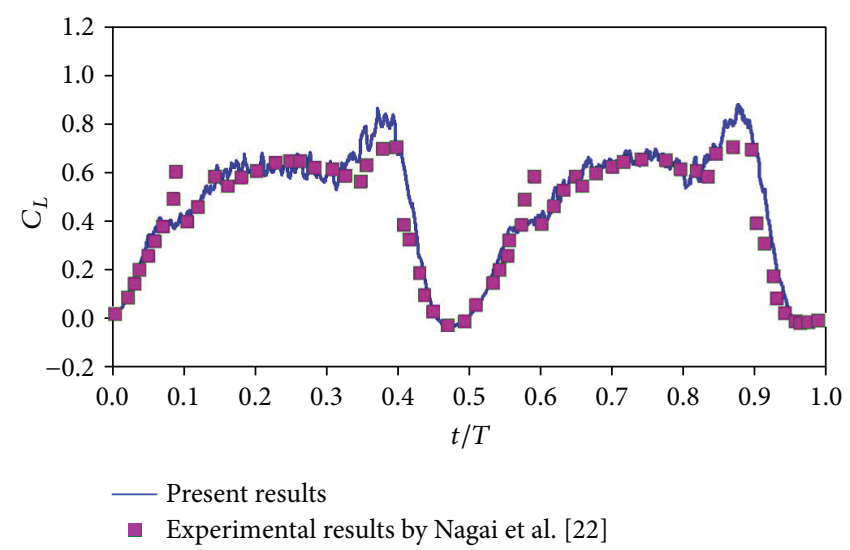

(b) Lift coefficient

Figure 6: Comparison of the drag and lift coefficient of the wing obtained by the present numerical method and literature experimental data.

for comparison. The $3 \mathrm{D}$ rigid and lumped flexibility bionic hovering wing are performed with $\theta_{m}=\pi / 3, f=$ $120 \mathrm{~Hz}$, and $\mathrm{Re}=2347$, and the parameters of the lumped flexibility wing are characterized by frequency ratio $f^{*}$ and density ratio $\rho^{*}$. The details of $f^{*}$ as well as $\rho^{*}$ on the lift and hovering efficiency of the wing will be analyzed in the following.

6.1. Varying Frequency Ratio. To study the effect of frequency ratio $f^{*}$ on the aerodynamic performance of the $3 \mathrm{D}$ lumped flexibility bionic hovering wing, we fix the density ratio $\rho^{*}=1000$, and the frequency ratio with $f^{*}=0.00,0.40$, $0.50,0.67,1.00,1.50$, and 2.00 are considered. Note that $f^{*}=0.00$ is represented as rigid wing, and as $f^{*}$ increases, the flexibility of the wing increases.

Figure 7 shows the performance of the 3D lumped flexibility wing with different $f^{*}$. It is seen from Figure 7(a) that the mean lift coefficients first increase as $f^{*}$ increases when $f^{*}$ is smaller than 1.00 and reach a maximum at $f^{*}=1.00$; however, they drop sharply when $f^{*}$ crosses over 1.00 , which indicates that the flexibility wing with $f^{*}=1.00$ has the best lift generation performance for the wing considered in this section.

It is clear in Figure 7(b) that the mean drag coefficients increase as $f^{*}$ increases when $f^{*}$ is smaller than 0.67 and reach a maximum value 0.00 at $f^{*}=0.67$; however, they drop sharply when $f^{*}$ crosses over 0.67 . It is also found in this figure that the mean drag coefficients have negative values expect for the wing with $f^{*}=0.67$, which indicates that the thrust are generated for the other flexible wings.

It is obvious in Figure $7(\mathrm{c})$ that the minimum mean energy coefficient is obtained when the flexible wing with $f^{*}=1.00$, and in comparison with the rigid wing, the mean energy coefficient of this wing is decreased by $36.27 \%$. However, for the other flexible wing, the frequency ratio $f^{*}$ has little effect on the mean energy coefficient; they have almost identical values.

It is seen from Figure $7(\mathrm{~d})$ that a similar variation trend of hovering efficiencies and lift coefficients with frequency ratio $f^{*}$ are observed. The energy efficiencies increase with the frequency ratio when the ratio is smaller than 1.00 and reach a maximum at $f^{*}=1.00$, which indicates that the best hovering performance of the wing is achieved when the flexible wing with $f^{*}=1.00$. However, they drop sharply when $f^{*}$ crosses over 1.00 and even a negative value is observed, which indicates that the hovering performance is deteriorated. This conclusion is different with the results obtained in our previous 2D lumped flexible wing study [27], at which we concluded that the best performance of the wing is achieved when the wing with frequency ratio $f^{*}$ is close to but less than 1.00 .

To analyze the mechanism of how the flexibility enhances the hovering performance of the lumped flexibility bionic wing details, Figure 8 shows the time variation of lift coefficients, drag coefficients, and energy coefficients of the above considered wings. It is obvious in this figure that after six flapping cycle simulations, periodic lift, drag, and energy coefficients have been established. It is also observed in this figure that the flexible wing with $f^{*}=1.00$ not only has smaller drag and energy coefficient amplitude than the other flexible wings but also has smoother time variation trend (with smaller curve slope), while for the lift coefficient, it has a larger amplitude, which is the reason why the flexible wing with $f^{*}=1.00$ has a larger mean lift and smaller energy coefficient as shown in Figure 7.

Figure 9 plots the time variation of passive pitching angle $\beta$ of the flexible wing with $f^{*}=1.00$ and 1.50 , and the time variation of flapping motion $\theta$ is also presented for comparison. It is obvious in this figure that $f^{*}$ influences $\beta$ significantly. For the flexible wing with $f^{*}=1.00$, the peak negative angle takes place at a phase angle of $0.86 \pi$, while for flapping angle $\theta$, the peak negative angle happens at a phase angle of $1.01 \pi$; moreover, the passive pitching angle $\beta$ of the flexible wing with $f^{*}=1.00$ also has a larger amplitude than the flapping angle $\theta$. While for the flexible wing with $f^{*}=1.50$, the passive pitching angle has visibly smaller amplitude than the flapping angle $\theta$, and the variation cycle of $\beta$ is two times that of the flapping motion $\theta$. Those findings could be useful to explain the phenomenon why the flexible wing with $f^{*}=$ 1.00 and 1.50 has different lift, drag, and energy coefficients as shown in Figure 8. 


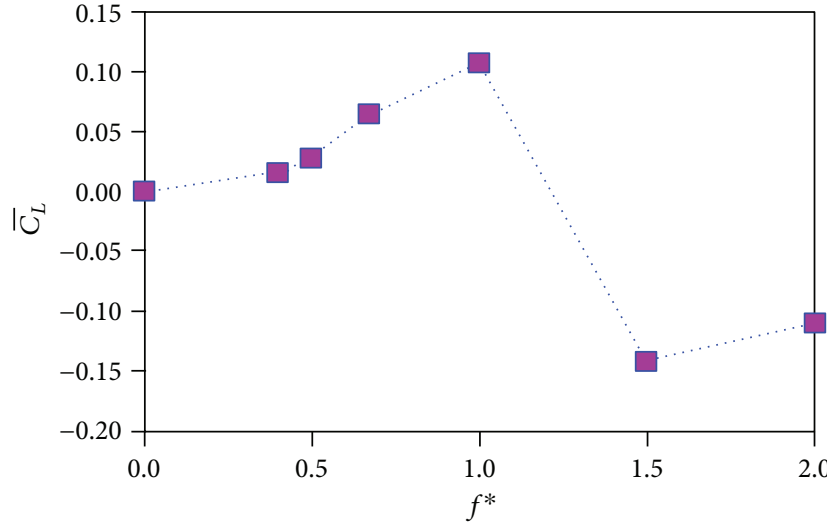

(a) Mean lift coefficients

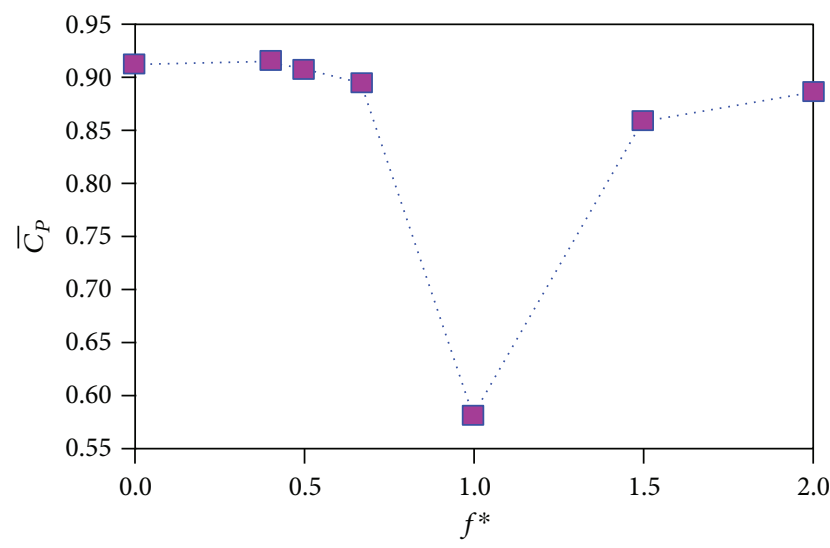

(c) Mean energy coefficients

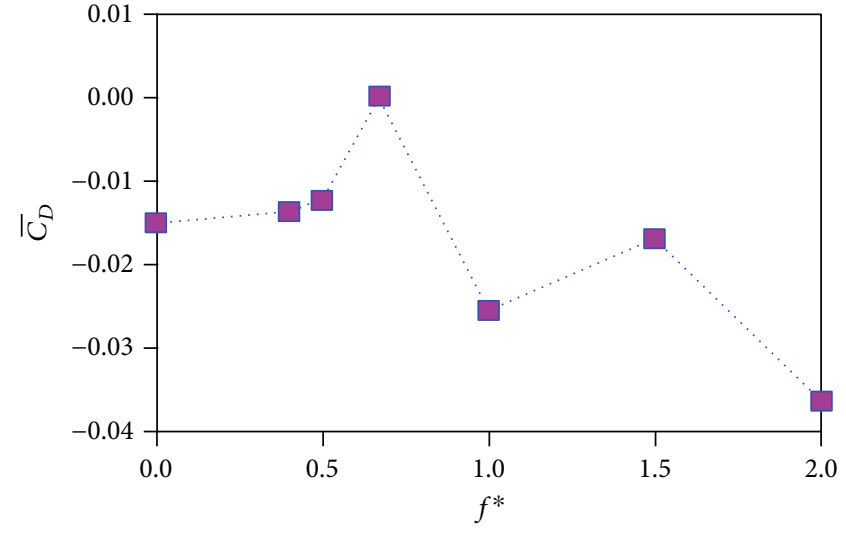

(b) Mean drag coefficients

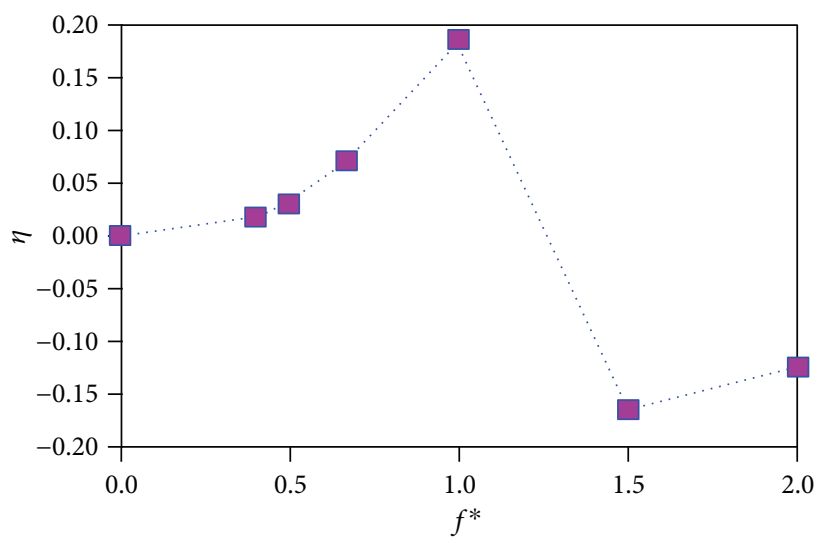

(d) Hovering efficiencies

Figure 7: The performance of the 3D lumped flexibility wing with different $f^{*}$.

In order to investigate why the flexible wing with $f^{*}=$ 1.00 and 1.50 possesses different performance with the rigid wing, Figures 10 and 11 show the vortex of the three considered wings at $t=11.30 T$ and $11.70 T$; for each time, three different wing span positions are investigated $(z=3.11 c$, $4.70 c$, and $6.29 c$ ). Obviously, it is seen in Figure 10 that similar vortex patterns near the wing surface are observed for the rigid wing and flexible wing with $f^{*}=1.00$ and 1.50; however, for the rigid wing and flexible wing with $f^{*}=1.50$, a visibly separated vortex is observed. It is also found in this figure that different vortex patterns are observed at wake of the three considered wings; a jet (in the negative $\mathrm{Y}$ direction) is observed for the flexible wing with $f^{*}=1.00$, while it is not for the rigid wing and flexible wing with $f^{*}=1.50$.

In Figure 11, a comparison with the rigid wing and flexible wing with $f^{*}=1.50$ is seen, in addition to the jet in the negative $\mathrm{Y}$ direction; the wake capture mechanism which is considered as the reason for the flapping wing having highlift generating capacity is also observed for the flexible wing with $f^{*}=1.00$, which is the reason why the lumped flexible wing with $f^{*}=1.00$ has better hovering performance than the rigid wing and flexible wing with $f^{*}=1.50$. It is also found in this figure that the flexible wing with $f^{*}=1.50$ has the weakest vortex near the wing surface, which leads the wing to have the worst aerodynamic performance.
6.2. Varying Density Ratio. To study the effect of density ratio $\rho^{*}$ on the aerodynamic performance of the 3D lumped flexibility bionic hovering wing, we fix the frequency ratio $f^{*}=1.00$ which has the best hovering performance of the studied wing in the last section, and the density ratio with $\rho^{*}=750,1000,1250,1500,1750$, and 2000 are considered.

Figure 12 plots the performance of the 3D lumped flexibility wing with different $\rho^{*}$. Three interesting phenomena can be concluded from this figure. Firstly, the density ratio has little effect on the mean drag coefficients, they have almost identical value at zero. Secondly, the mean energy coefficients of the wing monotonously decrease as $\rho^{*}$ increases. Thirdly, the maximum mean lift coefficient as well as hovering efficiency is achieved when the wing has $\rho^{*}=$ 1750 , which indicates that the flexible wing with $\rho^{*}=1750$ has better hovering performance than the other considered wing in this section. According to the study on the properties of insect wings by Combes and Daniel [28], the density ratio $\rho^{*}$ of insect wing is typically of the order $\mathrm{O}\left(10^{3}\right)$, which is consist with our findings.

In order to analyze the mechanism of how the density ratio influences aerodynamic performance of the 3D lumped flexibility bionic hovering wing, three typically density ratios $\rho^{*}=750,1750$, and 200 are considered.

Figure 13 shows the time variation of lift coefficients, drag coefficients, and energy coefficients of the above three 


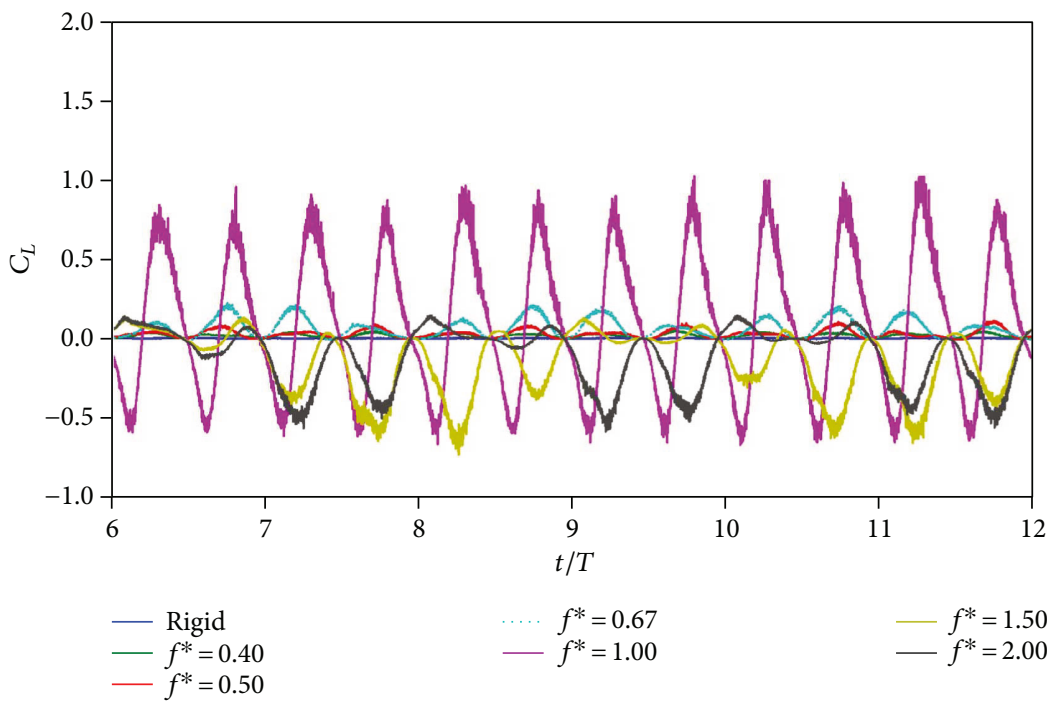

(a) Lift coefficients
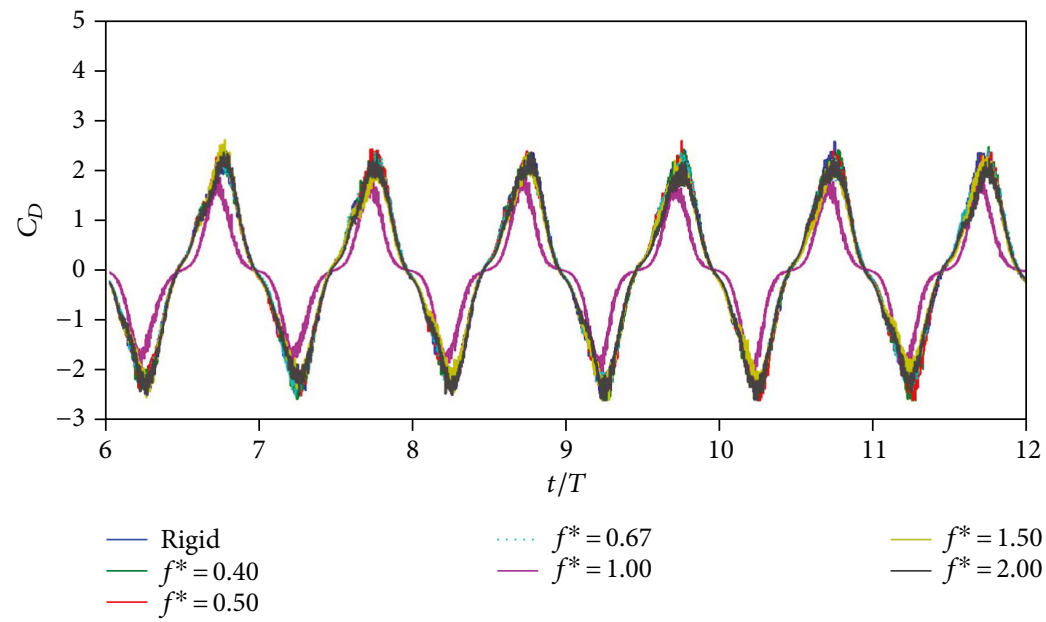

(b) Drag coefficients

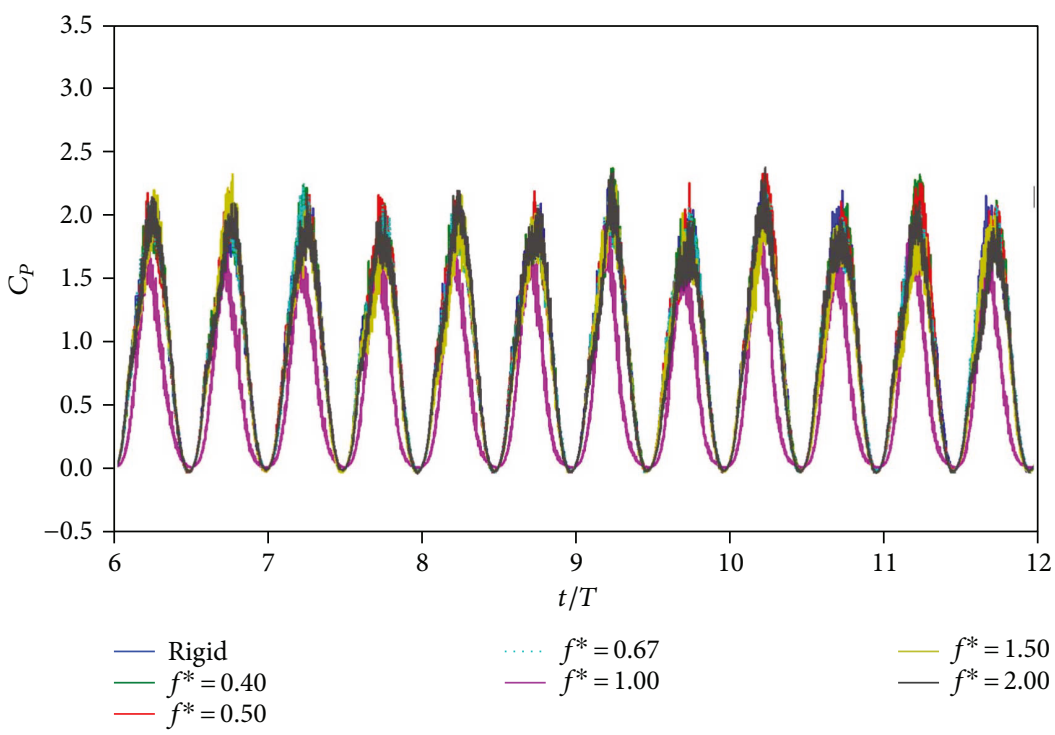

(c) Energy coefficients

FIGURE 8: Time variation of the lift coefficients, drag coefficients, and energy coefficients of the 3D lumped flexibility wing with different $f^{*}$. 


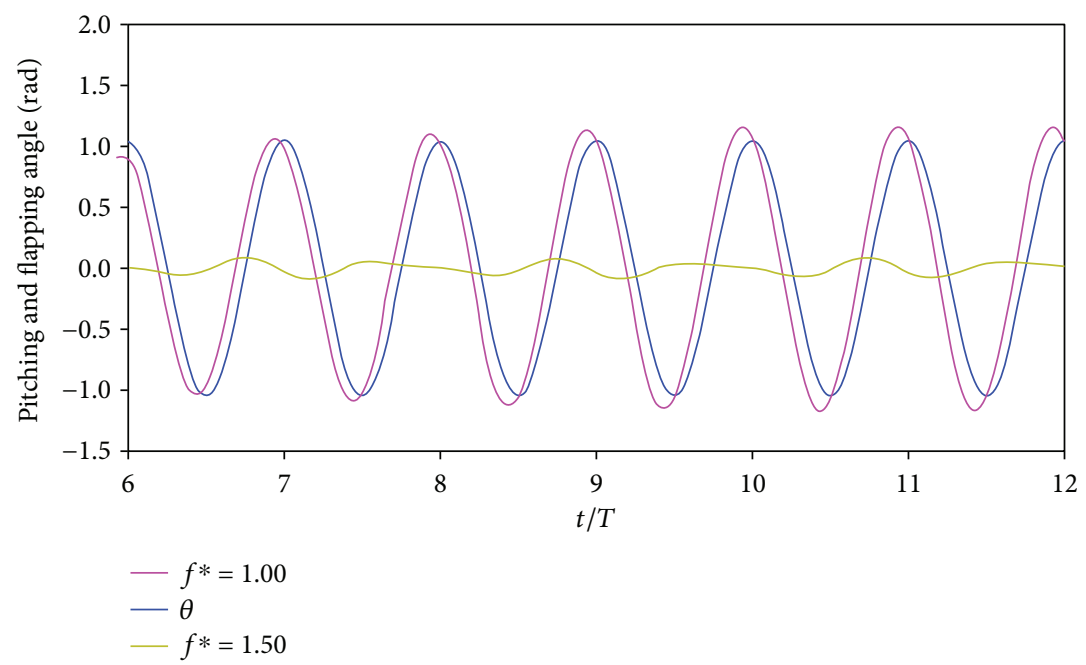

FIGURE 9: Time variation of passive pitching angle of flexible wings with $f^{*}=1.00$ and 1.50 and flapping angle $\theta$.
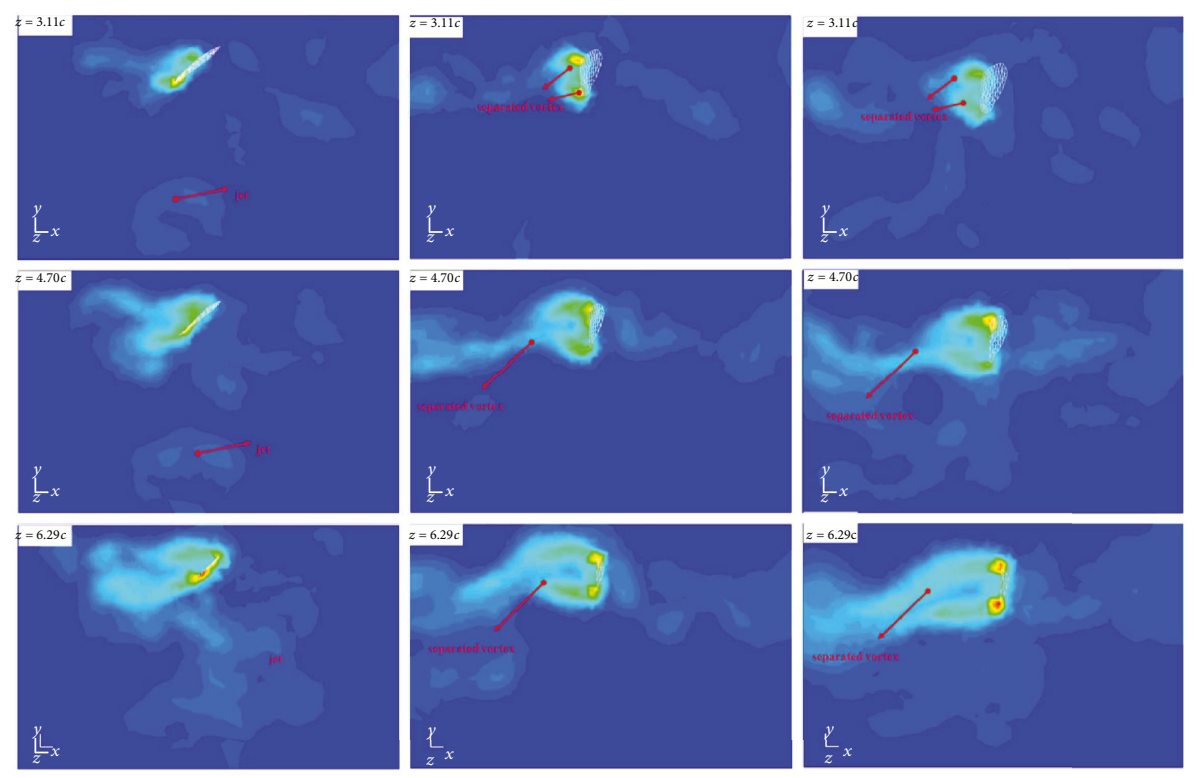

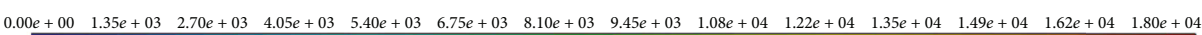

(a) Flexible wing with $f^{*}=1.00$

Contours of vorticity magnitude $(1 / \mathrm{s})$

(b) Rigid wing

(c) Flexible wing with $f^{*}=1.50$

Figure 10: The vortex of the rigid and flexible wing with $f^{*}=1.00$ and 1.50 at $t=11.30 T$ and $z=3.11 c, 4.70 c$, and $6.29 c$.

considered wings. Although a similar time variation trend of the lift coefficients are generated, there are phase differences between these three flexible wings, and the amplitude of the lift coefficient of the flexible wing with $\rho^{*}=1750$ is slightly larger than that of the flexible wing with $\rho^{*}=750$; on the contrary, the flexible wing with $\rho^{*}=2000$ results in the flexible wing with $\rho^{*}=1750$ to have larger mean lift coefficient and the flexible wing with $\rho^{*}=2000$ to have smaller mean lift coefficient, as shown in Figure 12(a). Obviously, the drag coefficient of the flexible wing with $\rho^{*}=1750$ and 2000 have almost identical values; however, the flexible wing with $\rho^{*}=750$ has larger amplitude almost during the whole flapping cycle; moreover, it also has rougher time variation trend, which indicates that the flexible wing with $\rho^{*}=1750$ and 2000 have better hovering stability. For the energy coefficient, the flexible wing with $\rho^{*}=2000$ has slightly smaller value than the flexible wing with $\rho^{*}=1500$ almost during the whole flapping cycle, while the flexible wing with $\rho^{*}=$ 750 has a larger value than the flexible wing with $\rho^{*}=1500$, which results in the mean energy coefficient of the flexible wing decreasing with $\rho^{*}$ increasing, as shown in Figure 12(c).

Figure 14 plots the time variation of passive pitching angle $\beta$ of the above three relation wings. It is clear in this figure that there are phase differences between these three 


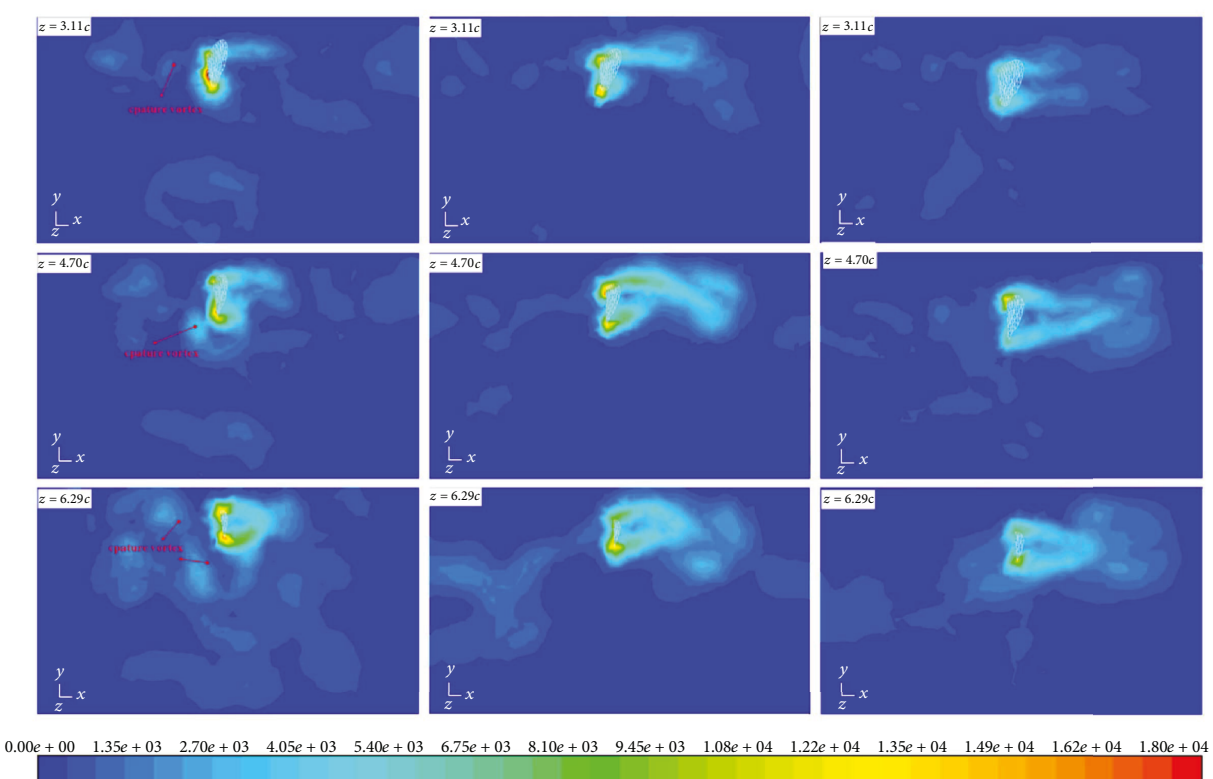
(a) Flexible wing with $f^{*}=1.00$
(b) Rigid wing
(c) Flexible wing with $f^{*}=1.50$

Figure 11: The vortex of the rigid and flexible wing with $f^{*}=1.00$ and 1.50 at $t=11.70 T$ and $z=3.11 c, 4.70 c$, and $6.29 c$.

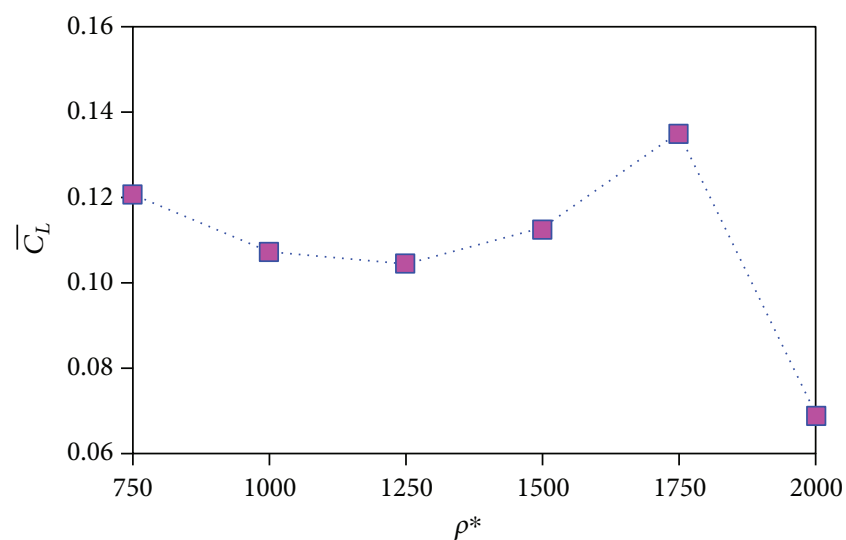

(a) Mean lift coefficients

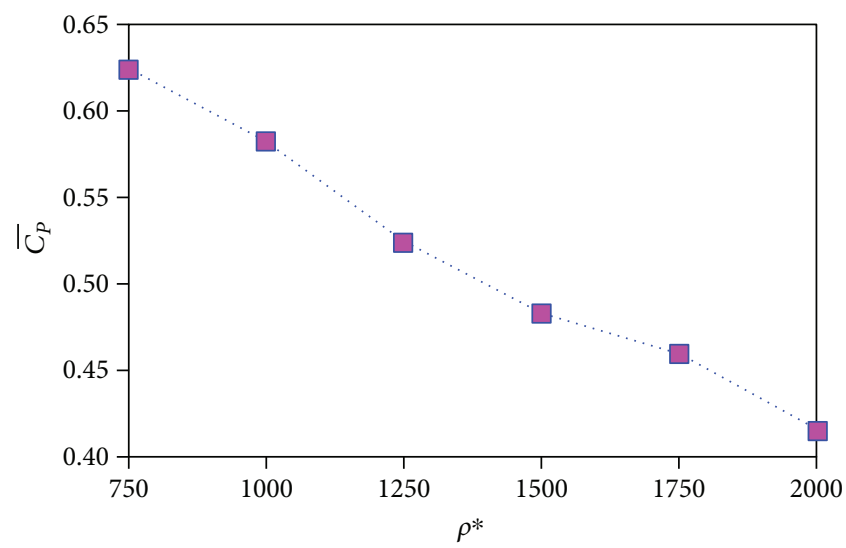

(c) Mean energy coefficients

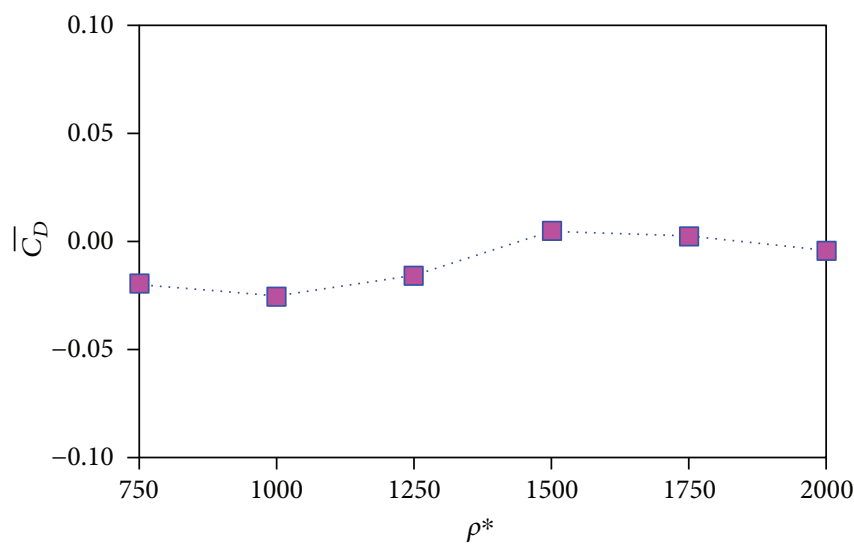

(b) Mean drag coefficients

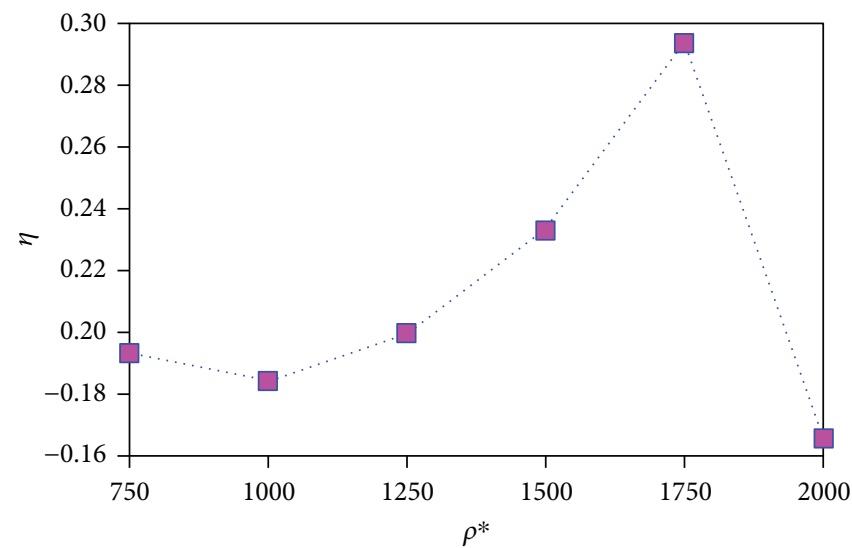

(d) Hovering efficiencies

Figure 12: The performance of the 3D lumped flexibility wing with different $\rho^{*}$. 

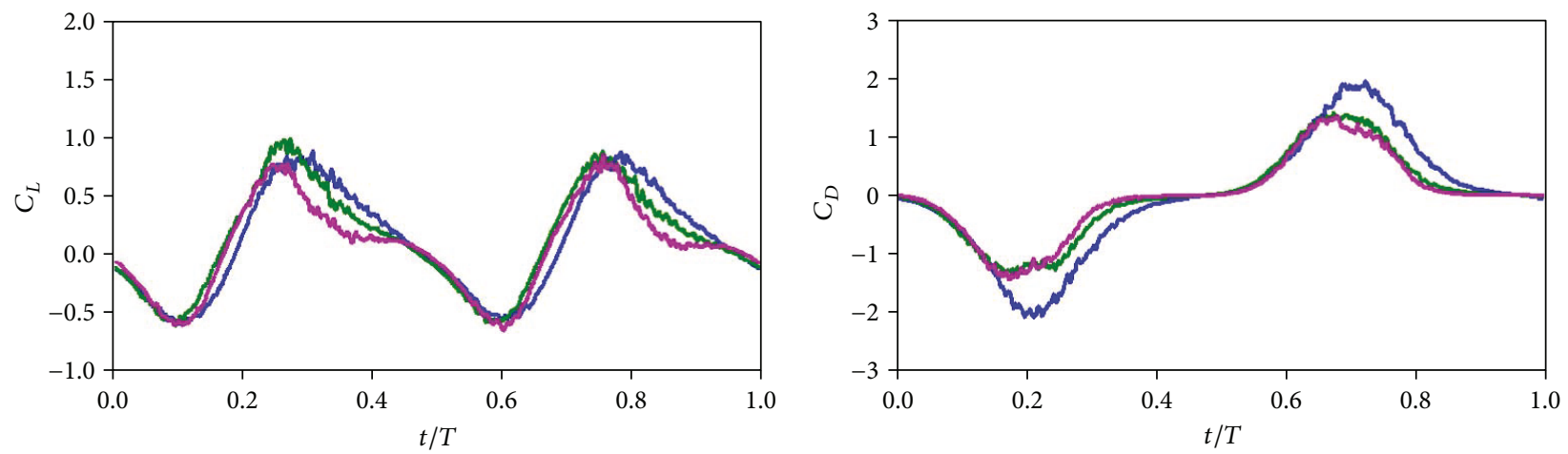

$$
\begin{aligned}
-\rho^{*} & =750 \\
\rho^{*} & =1750 \\
\rho^{*} & =2000
\end{aligned}
$$$$
-\rho^{*}=750
$$$$
-\rho^{*}=1750
$$$$
-\rho^{*}=2000
$$

(a) Lift coefficients

(b) Drag coefficients

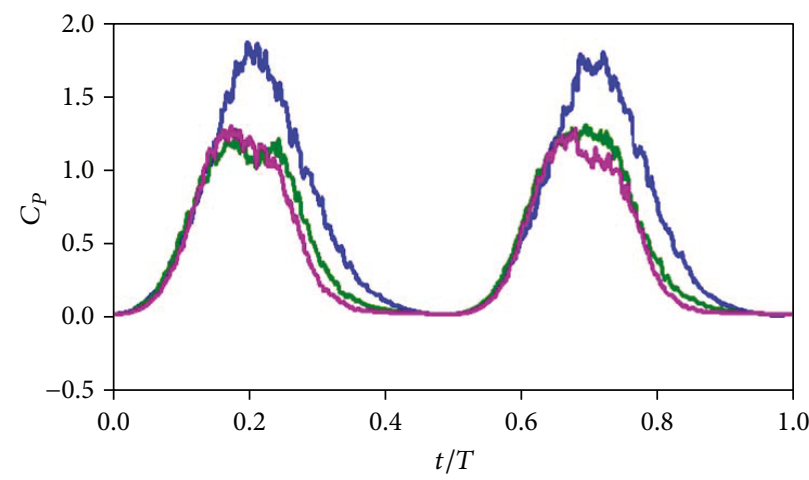

$$
\begin{aligned}
-\rho^{*} & =750 \\
\rho^{*} & =1750 \\
\rho^{*} & =2000
\end{aligned}
$$

(c) Energy coefficients

FIGURE 13: Time variation of the lift coefficients, drag coefficients, and energy coefficients of the 3D lumped flexibility wing with $\rho^{*}=750$, 1750, and 2000.

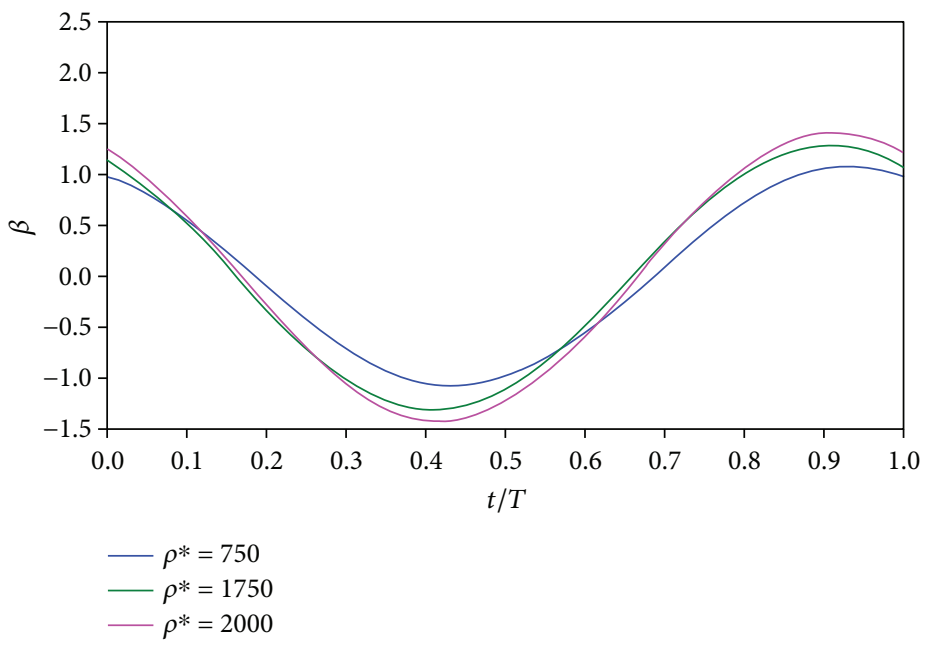

FIGURE 14: Time variation of passive pitching angle of flexible wings with $\rho^{*}=750,1750$, and 2000 .

angles, and the amplitude of $\beta$ increases with the increase in $\rho^{*}$, which may influence the vortex generated around the wing.
Figures 15 and 16 show the vortex of the flexible wing with $\rho^{*}=750,1750$, and 2000 at $t=0.30 T$ and $0.70 T$, and for each of time, again three different wing 


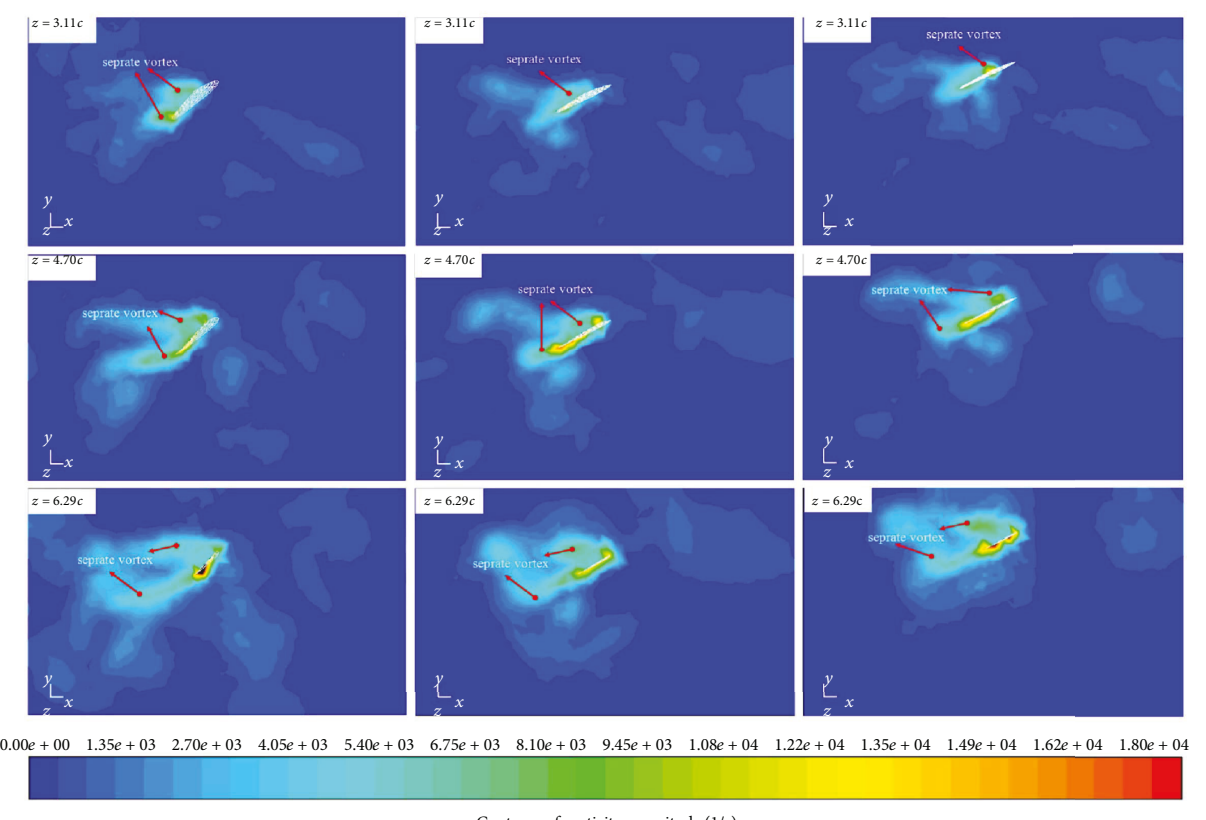
(a) $\rho^{*}=750$
(b) $\rho^{*}=1750$
(c) $\rho^{*}=2000$

FIGURE 15: The vortex of the flexible wing with $\rho^{*}=750,1750$, and 2000 at $t=0.30 T, z=3.11 c, 4.70 c$, and $6.29 c$.
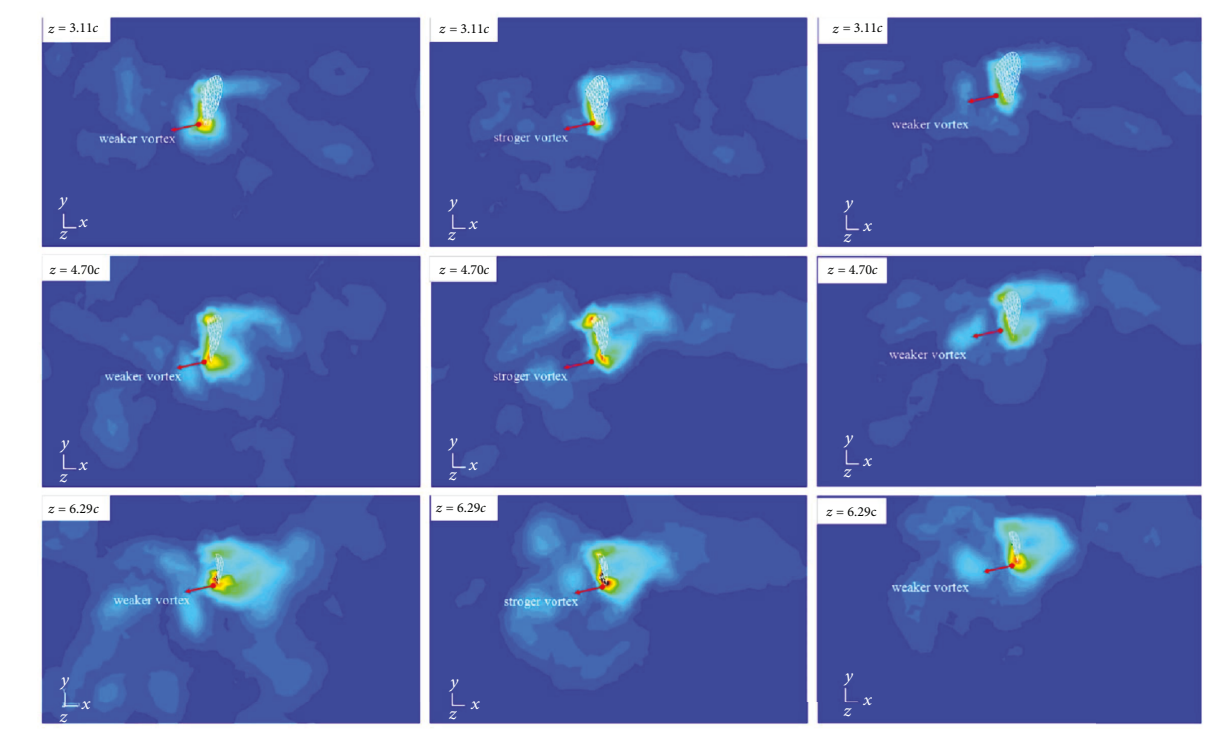

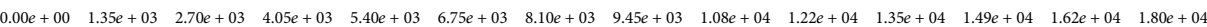

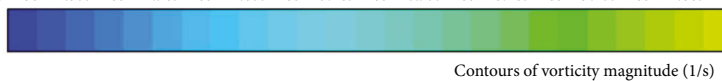
(a) $\rho^{*}=750$
(b) $\rho^{*}=1750$
(c) $\rho^{*}=2000$

FIGURE 16: The vortex of the flexible wing with $\rho^{*}=750,1750$, and 2000 at $t=0.70 T, z=3.11 c, 4.70 c$, and $6.29 c$.

span positions are investigated $(z=3.11 c, 4.70 c$, and $6.29 c)$. It is seen from Figure 15 that similar vortex patterns near the wing surface are observed for the three considered wings, from root to tip; the strength of the vortex is increased; however, the separate vortex of the flexible wing with $\rho^{*}=1750$ is closer to the wing surface than the other two flexible wings, which indicates that the flexible wing with $\rho^{*}=1750$ has an enhancement delay stall mechanism.

It is obvious in Figure 16 that the strongest vortex is generated around the flexible wing with $\rho^{*}=1750$. Moreover, complex vortex patterns are observed under the wake of the flexible wing with $\rho^{*}=750$, and the weakest vortex is generated around the flexible wing with $\rho^{*}=2000$, which 
indicates that the flexible wing with $\rho^{*}=750$ needs more energy to perform the wing hovering, and the flexible wing with $\rho^{*}=2000$ generates the smallest aerodynamic force, as shown in Figure 13.

\section{Conclusion and Future Work}

In this paper, a novel three-dimensional bionic wing which has lumped flexibility at the root is constructed; a numerical experiment is carried out to investigate the flexibility effects on the hovering performance of this novel bionic wing, where the incompressible Navier-Stokes (N-S) equations coupled with passive pitching motion by aerodynamic torque is solved. The flow field and aerodynamic forces are analyzed for a different frequency ratio $f^{*}$ and density ratio $\rho^{*}$ wings, and the results show that the $f^{*}$ and $\rho^{*}$ influence the hovering characteristics of the wing greatly. For the wing with different frequency ratio $f^{*}$, the best performance of the wing is achieved when the wing flaps at resonance $\left(f^{*}=1.00\right)$. For the wing with different density ratio $\rho^{*}$, it is found that the flexible wing with $\rho^{*}=1750$ has the best hovering performance, which agrees with the insect's density ratio in nature. In addition, delay stall and wake capture mechanic are observed around the wing with appropriate frequency ratio $f^{*}$ and density ratio $\rho^{*}\left(f^{*}=1.00, \rho^{*}=1750\right)$, therefore leading to better performance characteristics.

The results presented in this paper are based on lumped flexibility wing. Future work will simplify the bumblebee's wing as a $3 \mathrm{D}$ flexible plate.

\section{Conflicts of Interest}

The authors declare that they have no conflicts of interest.

\section{Acknowledgments}

This work was supported by the National Natural Science Foundation of China. (Project no. 51505347) and the Key Laboratory of Metallurgical Equipment and Control of Education Ministry, Wuhan University of Science and Technology Foundation (2015B07).

\section{References}

[1] F. Leys, D. Reynaerts, and D. Vandepitte, "Outperforming hummingbirds' load-lifting capability with a lightweight hummingbird-like flapping-wing mechanism," Biology Open, vol. 5, no. 8, pp. 1052-1060, 2016.

[2] V. M. Mwongera and Department of Aerospace Engineering, University of Bristol, UK, "A review of flapping wing MAV modelling," International Journal of Aeronautical Science \& Aerospace Research, vol. 2, no. 2, pp. 27-36, 2015.

[3] W. Shyy, H. Aono, S. K. Chimakurthi et al., "Recent progress in flapping wing aerodynamics and aeroelasticity," Progress in Aerospace Sciences, vol. 46, no. 7, pp. 284-327, 2010.

[4] M. Vanella, T. Fitzgerald, S. Preidikman, E. Balaras, and B. Balachandran, "Influence of flexibility on the aerodynamic performance of a hovering wing," Journal of Experimental Biology, vol. 212, no. 1, pp. 95-105, 2009.
[5] S. Shkarayev and R. Kumar, "Kinematics and inertial effects in locust flapping wings," Experimental Mechanics, vol. 56, no. 2, pp. 245-258, 2016.

[6] C. Zhang and C. Rossi, "A review of compliant transmission mechanisms for bio-inspired flapping-wing micro air vehicles," Bioinspiration \& Biomimetics, vol. 12, no. 2, article 025005, 2017.

[7] S. Tobing, J. Young, and J. C. S. Lai, "Effects of wing flexibility on bumblebee propulsion," Journal of Fluids and Structures, vol. 68, pp. 141-157, 2017.

[8] D. Ishihara, T. Horie, and M. Denda, "A two-dimensional computational study on the fluid-structure interaction cause of wing pitch changes in dipteran flapping flight," Journal of Experimental Biology, vol. 212, no. 1, pp. 1-10, 2009.

[9] D. Ishihara, T. Horie, and T. Niho, "An experimental and three-dimensional computational study on the aerodynamic contribution to the passive pitching motion of flapping wings in hovering flies," Bioinspiration \& Biomimetics, vol. 9, no. 4, article 046009, 2014.

[10] D. Ishihara and T. Horie, "Passive mechanism of pitch recoil in flapping insect wings," Bioinspiration \& Biomimetics, vol. 12, no. 1, article $016008,2016$.

[11] S. Michelin and S. G. Llewellyn Smith, "Resonance and propulsion performance of a heaving flexible wing," Physics of Fluids, vol. 21, no. 7, article 071902, 2009.

[12] H. Masoud and A. Alexeev, "Resonance of flexible flapping wings at low Reynolds number," Physical Review E, vol. 81, no. 5, article 056304, 2010.

[13] M. N. J. Moore, "Analytical results on the role of flexibility in flapping propulsion,” Journal of Fluid Mechanics, vol. 757, pp. 599-612, 2014.

[14] J. Zhu, C. Zhou, C. Wang, and L. Jiang, "Effect of flexibility on flapping wing characteristics under forward flight," Fluid Dynamics Research, vol. 46, no. 5, article 055515, 2014.

[15] J. Zhu and C. Zhou, "The aerodynamic performance of flexible wing in plunge," Journal of Mechanical Science and Technology, vol. 28, no. 7, pp. 2687-2695, 2014.

[16] Y. Chen, N. Gravish, A. L. Desbiens, R. Malka, and R. J. Wood, "Experimental and computational studies of the aerodynamic performance of a flapping and passively rotating insect wing," Journal of Fluid Mechanics, vol. 791, pp. 1-33, 2016.

[17] H. Cho, N. Lee, J. Y. Kwak, S. J. Shin, and S. Lee, “Threedimensional fluid-structure interaction analysis of a flexible flapping wing under the simultaneous pitching and plunging motion," Nonlinear Dynamics, vol. 86, no. 3, pp. 19511966, 2016.

[18] P. D. Yeh and A. Alexeev, "Effect of aspect ratio in freeswimming plunging flexible plates," Computers \& Fluids, vol. 124, pp. 220-225, 2016.

[19] R. J. Bomphrey, G. K. Taylor, and A. L. R. Thomas, "Smoke visualization of free-flying bumblebees indicates independent leading-edge vortices on each wing pair," Experiments in Fluids, vol. 46, no. 5, pp. 811-821, 2009.

[20] C. P. Ellington, "The aerodynamics of hovering insect flight. III. Kinematics," Philosophical Transactions of the Royal Society B: Biological Sciences, vol. 305, no. 1122, pp. 4178, 1984.

[21] C. P. Ellington, "The aerodynamics of hovering insect flight. IV. Aeorodynamic mechanisms," Philosophical Transactions of the Royal Society B: Biological Sciences, vol. 305, no. 1122, pp. 79-113, 1984. 
[22] H. Nagai, K. Isogai, T. Fujimoto, and T. Hayase, "Experimental and numerical study of forward flight aerodynamics of insect flapping wing," AIAA Journal, vol. 47, no. 3, pp. 730-742, 2009.

[23] A. R. Ennos, "The importance of torsion in the design of insect wings," Journal of Experimental Biology, vol. 140, no. 1, pp. 137-160, 1988.

[24] S. Camarri, M. V. Salvetti, B. Koobus, and A. Dervieux, "A low-diffusion MUSCL scheme for LES on unstructured grids," Computers \& Fluids, vol. 33, no. 9, pp. 1101-1129, 2004.

[25] T. Wang, C. G. Gu, B. Yang, and J. G. Huang, "PISO algorithm for unsteady flow field," Journal of Hydrodynamics, vol. 2, p. 14, 2003.

[26] J. Zhu, H. Huang, and H. Shen, "Self-starting aerodynamics analysis of vertical axis wind turbine," Advances in Mechanical Engineering, vol. 7, no. 12, 2015.

[27] C. Zhou and J. Zhu, "The aerodynamic performance of a 2D lumped flexible airfoil in forward flight," Proceedings of the Institution of Mechanical Engineers Part G: Journal of Aerospace Engineering, vol. 228, no. 8, pp. 1363-1374, 2014.

[28] S. A. Combes and T. L. Daniel, "Flexural stiffness in insect wings I. Scaling and the influence of wing venation," Journal of Experimental Biology, vol. 206, no. 17, pp. 2979-2987, 2003. 


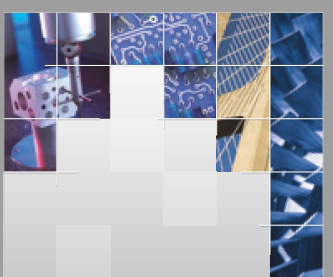

\section{Enfincering}
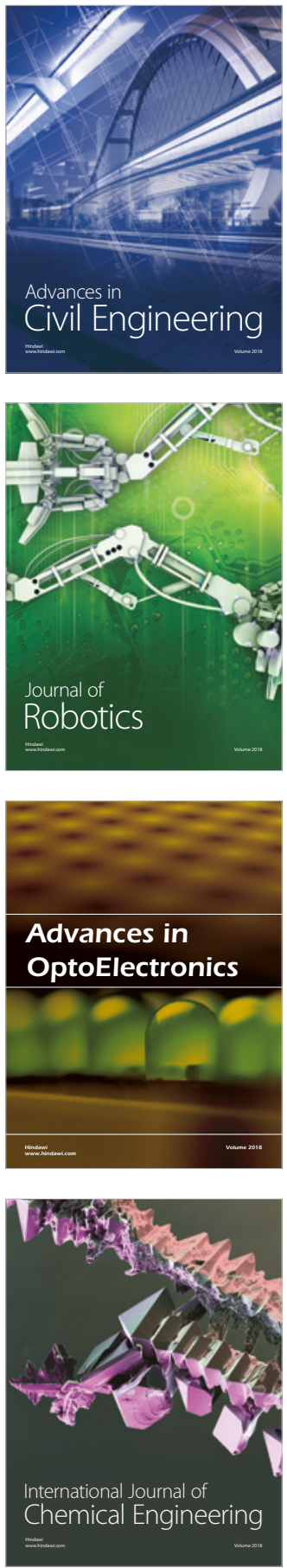

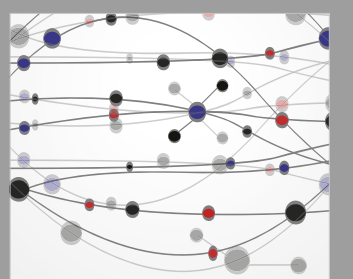

\section{Rotating \\ Machinery}

The Scientific World Journal

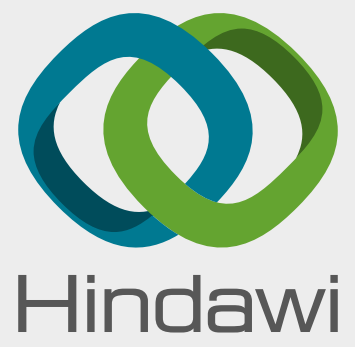

Submit your manuscripts at

www.hindawi.com
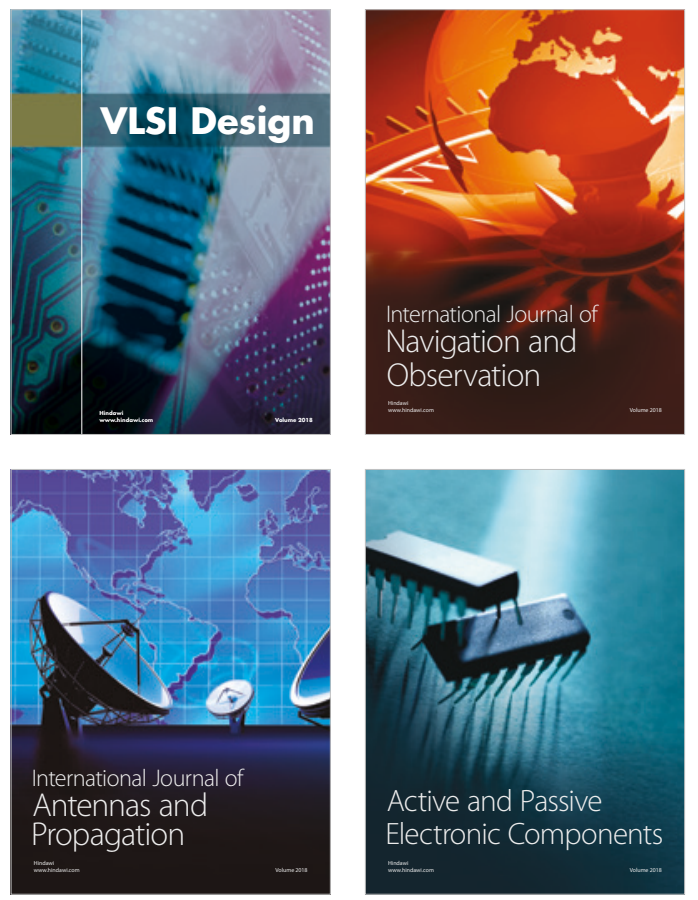
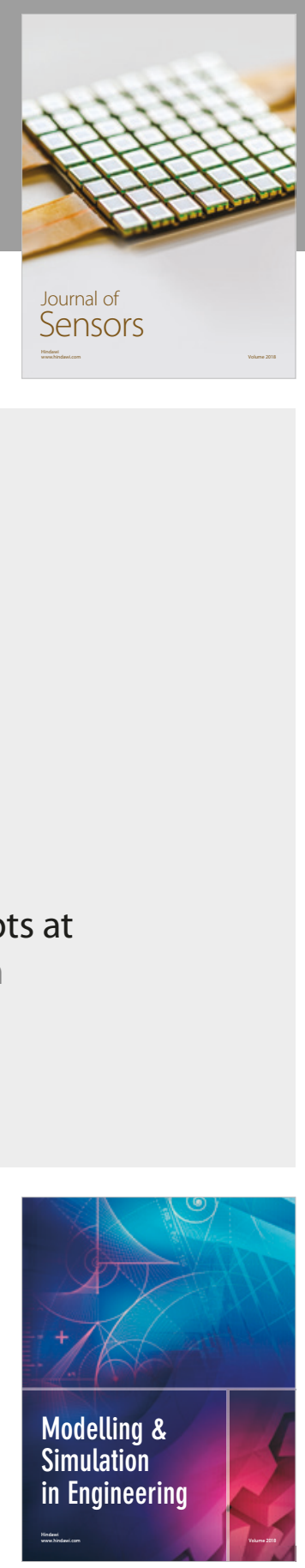

\section{Advances \\ Multimedia}
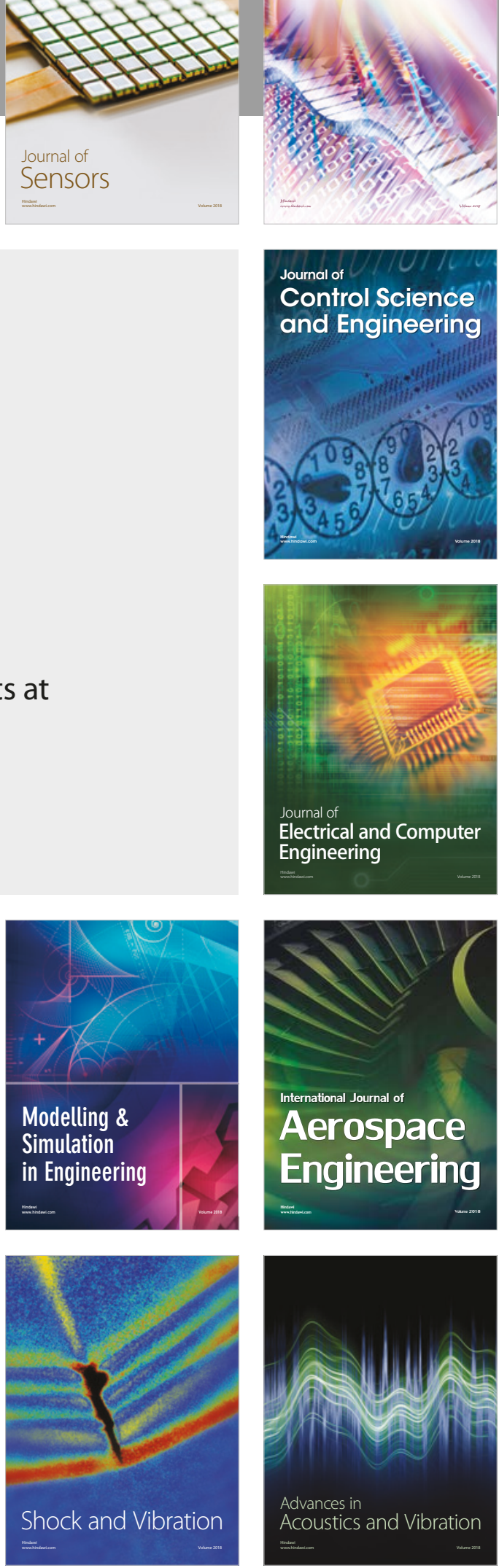